\title{
Efficient Diffractive Phase Optics for Electrons
}

\author{
Jordan Pierce ${ }^{1}$, Tyler Harvey ${ }^{1}$, Amit Agrawal $^{2}$, Peter Ercius ${ }^{3}$, Martin Linck $^{3}$, Benjamin J. McMorran ${ }^{4}$ \\ ${ }^{1 .}$ Department of Physics, University of Oregon, Eugene, Oregon \\ ${ }^{2}$ Center for Nanoscale Science and Technology, National Institute of Standards and Technology, \\ Gaithersburg, Maryland and Department of Electrical Engineering, Syracuse University, Syracuse, \\ New York \\ ${ }^{3}$ National Center for Electron Microscopy, Lawrence Berkeley National Laboratory, Berkeley, \\ California \\ ${ }^{4}$ Center for Nanoscale Science and Technology, National Institute of Standards and Technology, \\ Gaithersburg, Maryland and Department of Physics, University of Oregon, Eugene, Oregon
}

Traditional TEM electron beams are designed reproduce a plane wave inasmuch as this is possible. However, non-gaussian beam profiles can be useful for new forms of TEM and STEM techniques such as electron magnetic circular dichroism (EMCD) [1,2]. Central to this is the ability to reliably and efficiently create the desired waveform. For this we look at the use of phase gratings milled via FIB milling on a $20 \mathrm{~nm}-75 \mathrm{~nm}$ thick $\mathrm{Si}_{3} \mathrm{~N}_{4}$ membrane. Phase gratings, as opposed to amplitude gratings, allow for higher efficiency - greater than 30\% compared to $10.1 \%$. Phase diffraction gratings have been used in previous studies to create specified beam profiles such as Laguerre Gaussian modes [3], but there has been no comprehensive study of optimizing the grating profile. Diffraction gratings in general will create more than just the desired beam and optimizing the beam separation and efficiency can be difficult. For a $300 \mathrm{keV}$ beam the electrons have a deBroglie wavelength of $1.93 \mathrm{pm}$, which, for an 80 $\mathrm{nm}$ pitch grating, corresponds to a $25 \mu \mathrm{rad}$ first order diffraction angle. Optimizing the phase gratings consists of four main parameters - intensity of in the desired mode relative to the incident intensity (absolute efficiency), intensity of the desired mode relative to the total transmitted intensity (relative efficiency), spatial coherence of the grating, and minimizing the pitch of the grating.

Using silicon nitride for the phase grating material is a good choice because it is mechanically quite robust and has a mostly constant low electron scattering cross section. As the electron beam interacts with the membrane, two general interactions occur - elastic scattering and inelastic scattering. The elastic scattering is a function of the mean inner potential. For silicon nitride, a groove depth of about 34 $\mathrm{nm}$ will maximize the first order relative efficiency, so the membrane should be at least $50 \mathrm{~nm}$ thick. For an unmodulated membrane, a thickness of $20 \mathrm{~nm}$ will inelastically scatter $\sim 20 \%$ of the incident beam, while for a thickness of $75 \mathrm{~nm}$ this becomes $\sim 50 \%$.

For this study, we created linear diffraction gratings using a $30 \mathrm{keV} \mathrm{Ga}{ }^{+}$beam with a nominal beam current of between $1.5 \mathrm{pA}$ and $12.7 \mathrm{pA}$. We found that milling the patterns in a single-pass manner generally results in unacceptable spatial coherence. This can be rectified by milling with multiple identical passes $(10-100)$. Figure 2 shows several $10 \mu \mathrm{m}$ gratings milled into a $75 \mathrm{~nm}$ thick silicon nitride membrane. To achieve high efficiency several factors must be in place - the sample and beam must be stable for a relatively long time (several hours for large gratings) and the membrane must be sufficiently stiff to avoid stretching. We developed a reliable method for creating gratings with a pitch of 80-100 nm and a diameter of 30 microns or less. Figure 3 shows the AFM profile for several different gratings. To model the diffraction, we assumed that the FIB milled trenches are gaussian in profile and that there is no interplay between the implanted Gallium and the mean inner potential. With this, and the 
AFM measured open fraction of the gratings, we can predict how efficient they should be. Figure 1 shows an overview of the relative first order diffraction efficiency of more than 30 gratings.

\section{References:}

[1] J Verbeeck, H Tian and P Schattschneider, Nature, 467 (2010), p. 301 - 304

[2] B McMorran et al., Science 331 (2011), p. 192 - 195

[3] B. J. Mcmorran et al, Electron vortex beams with high quanta of orbital angular momentum (2012).
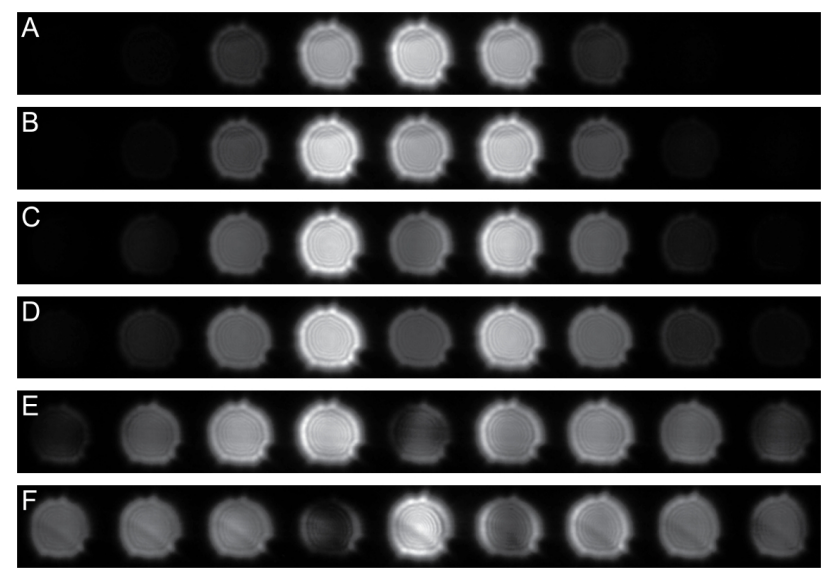

Figure 2: TEM diffraction images of several gratings of varying depth. Each grating was milled with a $30 \mathrm{keV} \mathrm{Ga}{ }^{+}$beam at $9.7 \mathrm{pA}$. The membrane is $75 \mathrm{~nm}$ thick silicon nitride coated with a thin layer of platinum. Each grating is 10 microns in diameter. The depth of the grooves increases from $A(\sim 30 \mathrm{~nm})$ to $F(\sim 50 \mathrm{~nm})$. These images are taken out of focus to emphasize the spatial coherence of the beams.

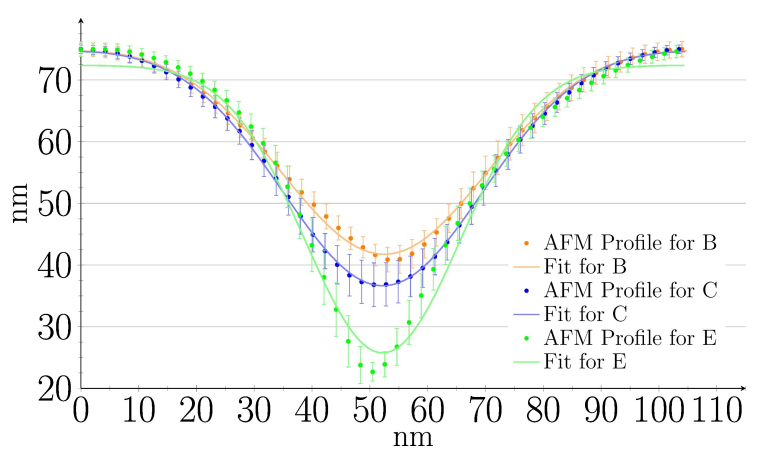

Figure 3: Average AFM groove shape of a 1 $\mu \mathrm{m}^{2}$ section from the labeled gratings. $A$ best fit periodic gaussian fit is also plotted for each grating.

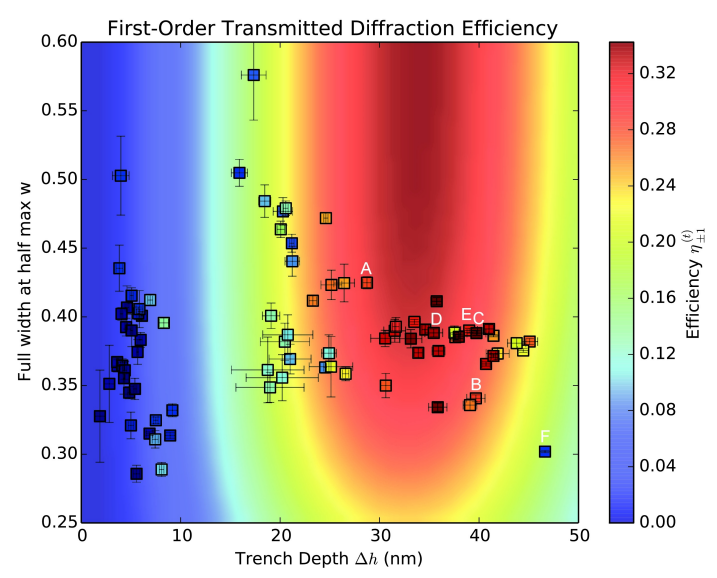

Figure 1: First order relative diffraction efficiency for a large number of gratings. The background color map are the simulated values based on the periodic gaussian shape for the grooves. 